\title{
The correlation of emphysema or airway obstruction with the risk of lung cancer: a matched case-controlled study
}

\author{
K. Kishi*, J.W. Gurney", D.R. Schroeder", P.D. Scanlon ${ }^{+}$, S.J. Swensen" ${ }^{\#}$ J.R. Jett ${ }^{+}$
}

The correlation of emphysema or airway obstruction with the risk of lung cancer: a matched case-controlled study. K. Kishi, J.W. Gurney, D.R. Schroeder, P.D. Scanlon, S.J. Swensen, J.R. Jett. C ERS Journals Ltd 2002.

ABSTRACT: A matched case-controlled study was conducted to determine if airway obstruction or emphysema were associated with an increased risk of lung cancer.

Lung cancer cases $(n=24)$ were identified through a low-dose spiral computed tomography (CT) screening trial from 1,520 participants. Four controls without lung cancer were selected for each case from the participants and matched by sex, age and smoking history. Emphysema was assessed by quantitative CT analysis. Conditional logistic regression was employed to assess results of spirometry and CT quantitative analysis as potential risk factors for lung cancer.

The likelihood of lung cancer was found to be significantly increased for those with forced expiratory volume in one second (FEV1) $\leqslant \mathbf{4 0} \%$ of predicted. The results suggested that a lower percentage of predicted FEV1 was indicative of lung cancer. No compelling evidence was found to suggest that the percentage of emphysema was associated with lung cancer.

These results suggest an increased risk of lung cancer associated with airway obstruction. However, percentage of emphysema as determined by computed tomography was not associated with an increased risk of lung cancer. Eur Respir J 2002; 19: 1093-1098.
*Division of Respiratory Diseases, Toranomon Hospital, Tokyo, Japan. ${ }^{\#}$ Dept of Radiology, Dept of Health Sciences Research and Division of Pulmonary Medicine, Mayo Clinic, Rochester, MN, USA.

Correspondence: K. Kishi, Toranomon Hospital, 2-2-2 Toranomon, Minatoku, Tokyo 105-8470, Japan. Fax: 81335827068

E-mail: kazumak@toranomon.gr.jp

Keywords: Airway obstruction emphysema lung cancer

quantitative computed tomography analysis

Received: July 262001

Accepted after revision February 21 2002
Lung cancer is the number one cause of death from cancer in the USA [1]. Approximately $85 \%$ of lung cancer occurs in current or former smokers. The risk of lung cancer increases with age and amount of smoke exposure [2]. Chronic obstructive pulmonary disease (COPD) is defined as a disease state characterized by the presence of airflow obstruction due to chronic bronchitis or emphysema [3]. Overall, smoking accounts for an estimated $80-90 \%$ of the risk of developing COPD [4]. Lung cancers frequently occur in patients with COPD [5]. Although cigarette smoke is the common aetiological factor for both lung cancer and COPD, several studies have shown that airway obstruction is associated with a four- to six-fold increased risk of lung cancer independent of smoking history [6, 7].

Emphysema is defined anatomically as abnormal permanent enlargement of the airspaces distal to the terminal bronchioles, accompanied by destruction of their walls without obvious fibrosis [3]. Recent studies have reported the detection of asymptomatic lung cancers in $2-5 \%$ of patients with severe emphysema who were being evaluated for lung volume reduction surgery $[8,9]$. These lung cancers were located peripherally in the lung and detected by computed tomography (CT) scans of the chest, which were performed as part of the pre-operative evaluation. Nevertheless, the relationship between the degree of emphysema, as measured by CT, and the risk of lung cancer has not been clearly defined.

Because the definition of emphysema is based on the anatomical demonstration of destruction of lung tissue, CT is well suited for in vivo identification [10]. CT and particularly, high-resolution CT (HRCT) detects emphysema with a greater sensitivity than chest radiography or pulmonary-function tests. In addition to being evaluated visually, emphysematous changes on CT can be quantified objectively by measuring lung attenuation $[11,12]$. Lung attenuation values in normal subjects range from -770-875 Hounsfield units (HU) [13]. The density in emphysematous lung is low, and voxels with low attenuation values can be highlighted by the use of "density mask" software [11]. Quantitative CT analysis using the "density-mask" technique has been shown to correlate well with the pathological assessment of emphysema. Recently, this technique has been applied to three dimensional (3D) volumetric reconstructions from spiral CT scans to assess lung volume and quantitation of emphysema [14, 15].

In 1999, investigators at Mayo Clinic launched an early lung cancer screening trial using low-dose spiral CT scan supplemented by sputum cytology and spirometry [16]. Using the data acquired from this trial, a matched case-controlled study was performed to determine if airways obstruction measured by 
spirometry or emphysema assessed by quantitative CT were associated with an increased risk of lung cancer in the screened cohort.

\section{Materials and methods}

\section{Study subjects}

From January 1-December 31 1999, 1,520 participants $(52 \%$ males) were entered into the low-dose spiral CT screening trial for detecting lung cancer at Mayo Clinic, Rochester. Males and females, aged $\geqslant 50$ yrs with a life expectancy of at least $5 \mathrm{yrs}$, who were current or former smokers (having quit $<10$ yrs previously) of $\geqslant 20$ pack-yrs, and who did not use supplemental oxygen were recruited. Candidates with a history of cancer within 5 yrs other than nonmelanoma skin cancer, in situ cervical cancer, or localized prostate cancer were excluded. The participants were recruited from respondents to advertisements and articles published in the lay press and from television reports.

The study protocol was approved by the Institutional Review Board and written informed consent was obtained. Initial studies included a low-dose spiral CT scan of the chest, induced sputum cytology and spirometry (prevalence screening). By protocol, all participants will be retested yearly for 3 yrs (incidence screening). This report is based on cases of lung cancer detected by January 52001 after 1 yr of follow-up.

Among 1,520 participants, 24 cases have been diagnosed with lung cancer to date. Of 24 cases 22 were detected by prevalence screening and two cases were detected by incidence screening. The controls were selected from participants without lung cancer. A 1:4 matched set design was employed whereby each lung cancer case was matched to four controls. Matching variables included sex, age, and pack-yrs of smoking with optimal matched sets identified using the approach described by RosEnBAUM [17] and Bergstralh and Kosanke [18].

\section{Computed tomography scan}

CT scans were performed with a High Speed Advantage CT scanner (General Electric Medical Systems, Milwaukee, WI, USA) in the helical mode without contrast material. The technical scan parameters included the following: 120 kilovolt peak $(\mathrm{kVp}), 40$ milliamperes $(\mathrm{mA}), 5 \mathrm{~mm}$ collimation with $3.5 \mathrm{~mm}$ reconstruction interval, $30 \mathrm{~mm} \cdot \mathrm{s}^{-1}$ table speed and $2: 1$ pitch. The CT images of the entire lung region were obtained in a single breath hold at full inspiration. Lung images were reconstructed with a highfrequency, edge-enhancing algorithm.

\section{Quantitative computed tomography analysis}

The 3D images of the lungs were reconstructed with the Advantage Windows 3D Analysis Package (General Electric Medical Systems). The threshold limits of
-700--1,024 HU were applied to exclude soft tissue surrounding the lung and large vessels within the lung. The 3D images were viewed as a shaded surface display at multiple angles to ensure the model was valid (fig. 1). The trachea, main-stem bronchi, and gastrointestinal structures were selectively removed from the model.

A histogram display of the $3 \mathrm{D}$ images showed the volume, attenuation distribution and SD of attenuation. The histogram provided a frequency distribution of voxels with specific attenuation numbers (in HU) in the lung. The volume of voxels with attenuation values $<-900 \mathrm{HU}$ were measured on the histogram. The -900 HU value was a threshold previously reported to separate emphysematous lung from normal lung [10, 15]. The volume of low attenuation voxels was then divided by the total lung volume to obtain the percentage of emphysema.

\section{Spirometry}

Spirometry was performed with a Puritan Bennett Renaissance pneumotach-based flow spirometer (Mallinckrodt, St Louis, MO, USA) according to the standards of the American Thoracic Society [19]. Forced expiratory volume in one second (FEV1) was expressed as $\%$ of predicted using the reference equations of CRAPO et al. [20].

\section{Statistical analysis}

All analyses were performed using, the 1:4 matched set of 24 cases and 96 controls. Conditional logistic regression was employed to assess whether \% pred FEV1, the ratio of FEV1 to forced vital capacity (FEV1/FVC), or the percentage of emphysema, as determined by the quantitative CT analysis are risk factors for lung cancer [21]. Since cases and controls

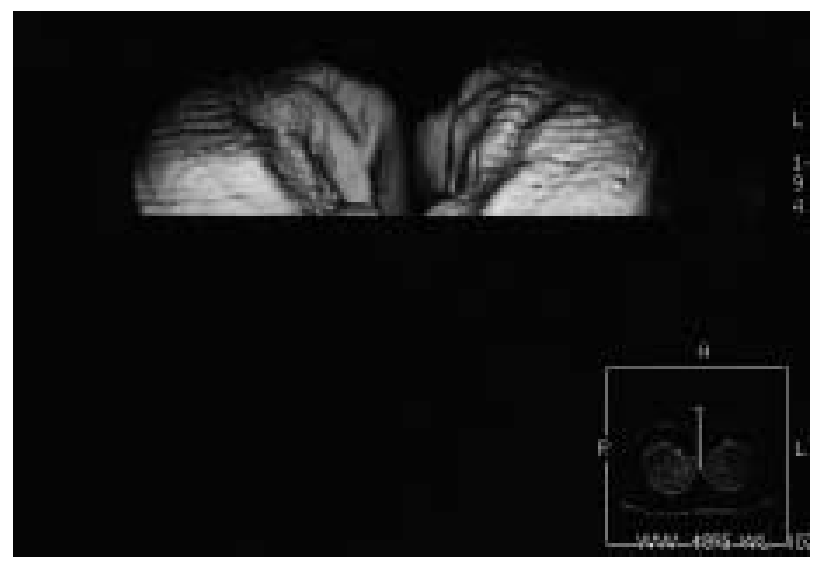

Fig. 1. - Volume reconstruction of the upper one-third of the lung. Axial images were constructed using three-dimensional volume rendering. From the volume reconstruction, the computer can calculate total lung volume or volume of an emphysematous lung. The trachea has been deliberately excluded from the reconstruction. The major airways to the segmental bronchi have been excluded as the computer cannot distinguish between normal dead space air and emphysematous lung. 
were matched for age, sex, and pack-yrs of smoking history, the use of the conditional logistic regression adjusts for these variables. Neither current-smoking status nor length of abstinence from smoking were considered in the matching procedure. Therefore, the duration of abstinence from smoking was calculated for each individual and included as a covariate in the conditional logistic regression analysis. For current smokers the duration of abstinence from smoking was assigned a value of zero. Per cent pred FEV1 was analysed as a continuous variable and also as a categorical variable using the categories $\geqslant 81 \%$, $61-80 \%, 41-60 \%$, and $\leqslant 40 \%$ [22]. FEV $1 / \mathrm{FVC}$ was analysed as a continuous variable and also as a categorical variable using the categories $\geqslant 71 \%, 61-70 \%$, $51-60 \%$, and $\leqslant 50 \%$. Percentage of emphysema, as determined by the quantitative CT analysis, was analysed as a continuous variable and also as a categorical variable using the categories $0-4 \%, 5-9 \%$, $10-14 \%$, and $\geqslant 15 \%$. Odds ratios (OR) with corresponding $95 \%$ confidence intervals $(\mathrm{Cl})$ were calculated where appropriate. In all cases, two-sided tests were used with $\mathrm{p} \leqslant 0.05$ considered statistically significant.

\section{Results}

There were 24 cases (10 males, 14 females) and 96 controls (40 males, 56 females) (table 1). Cases and controls were matched (1:4) for sex, age, and pack-yrs of smoking history. The mean \pm SD age was $63.7 \pm$ 6.9 yrs for the cases and $63.2 \pm 6.3$ yrs for the controls. Age was matched within \pm 3 yrs of the case for $97 \%$ (93 of 96) of the controls. The mean smoking history was $59 \pm 15$ pack-yrs for cases and $58 \pm 14$ pack-yrs for controls. Pack-yrs of smoking history were matched within \pm 5 pack-yrs of the case for $90 \%$ (86 of 96) of the controls and within \pm 10 pack-yrs of the case for $97 \%$ (93 of 96) of the controls. Eleven of $24(46 \%)$ cases and 55 of $96(57 \%)$ controls were current smokers; and four of $24(17 \%)$ cases and 14 of $96(15 \%)$ controls had abstained from smoking for $>5$ yrs.

The cell types of the 24 lung cancer cases included small cell carcinoma in three, squamous cell carcinoma in six, adenocarcinoma in 14 (including two with bronchoalveolar carcinoma), and large cell carcinoma in one. There were 12 cancers each in the right and left lung. Ten of $24(42 \%)$ lung cancers occurred in the upper lobes, one in the middle lobe, $12(50 \%)$ in the lower lobes and one in both middle and lower lobes. Of the 21 nonsmall cell lung cancer cases, 14 $(67 \%)$ were stage I, five $(24 \%)$ were stage II, and two $(10 \%)$ were stage IIIA at the time of diagnosis. All three small cell lung cancers were limited stage disease.

Conditional logistic regression was employed to assess results of spirometry and CT quantitative analysis as potential risk factors for lung cancer. The findings of these analyses are presented in table 2 . When analysed as a continuous variable, the results suggested that a lower percentage of FEV1 \% pred was predictive of lung cancer $(\mathrm{OR}=1.2$, for each $10 \%$ point decline, 95\% confidence interval (CI) 1.0-1.5,
Table 1. - Patient demographics"

\begin{tabular}{lcc}
\hline & Controls & Cases \\
\hline Subjects n & 96 & 24 \\
Gender \% & 58 & 58 \\
$\quad$ Female & 42 & 42 \\
$\quad$ Male & & \\
Age yrs & $63.2 \pm 6.3$ & $63.7 \pm 6.9$ \\
$\quad$ Mean \pm SD & $54.0-79.0$ & $55.0-79.0$ \\
$\quad$ Range & $57.8 \pm 14.4$ & $59.0 \pm 15.2$ \\
Pack-yrs of smoking & $35.0-104.0$ & $36.0-100.0$ \\
$\quad$ Mean \pm SD & 57 & \\
$\quad$ Range & 28 & 46 \\
Smoking Status \% & 15 & 17 \\
$\quad$ Current & & \\
$\quad$ Former $<5$ yrs & $1.5 \pm 2.6$ & $1.9 \pm 2.9$ \\
$\quad$ Former $\geqslant 5$ yrs & $0-9.4$ & $0-8.6$ \\
Duration of abstinence yrs & Mean \pm SD & \\
$\quad$ Range &
\end{tabular}

\#: cases and controls were matched 1:4 for sex age and packyrs; ${ }^{\uparrow}$ : duration of abstinence from smoking was assigned a value of zero for current smokers.

$\mathrm{p}=0.082$ ). When treated as a categorical variable, the likelihood of lung cancer was significantly increased for those with FEV1 $\leqslant 40 \%$ pred $(\mathrm{OR}=9.6,95 \% \mathrm{CI}$ 1.5-60.1 relative to those with $\mathrm{FEV} 1>80 \%$ pred, $\mathrm{p}=0.016$ ). There was also evidence that suggested lower $\mathrm{FEV} 1 / \mathrm{FVC}$ was predictive of lung cancer $(\mathrm{p}=0.083)$ and that those with $\mathrm{FEV} 1 / \mathrm{FVC} \leqslant 50 \%$ pred were at increased risk $(\mathrm{OR}=4.1,95 \%$ CI $1.0-17.2$ relative to those with $\mathrm{FEV} 1 / \mathrm{FVC}>70 \%$ pred, $\mathrm{p}=0.056$ ). No evidence was found to suggest that the percentage of emphysema, as determined by the quantitative CT analysis, was associated with lung cancer $(\mathrm{OR}=1.1$, for each $10 \%$ point increase, $95 \% \mathrm{CI}$ $0.6-1.9, \mathrm{p}=0.763)$.

\section{Discussion}

The results of this study indicate an increased risk of lung cancer associated with airway obstruction independent of smoking history. There was evidence suggesting that both lower percentage of FEV1\% pred $(\mathrm{OR}=1.2,95 \%$ CI $1.0-1.5)$ and $\mathrm{FEV} 1 / \mathrm{FVC}(\mathrm{OR}=1.4$, $95 \%$ CI 1.0-2.2) were predictive of lung cancer. The authors found that severe airway obstruction, with an FEV $1 \leqslant 40 \%$ pred, was significantly associated with a markedly increased risk of lung cancer $(\mathrm{OR}=9.6,95 \%$ CI 1.5-60.1). These data are consistent with previous prospective studies that assessed the risk of lung cancer in patients with airway obstruction [6, 7]. SKILLRUd et al. [6] studied 113 subjects with an FEV1 of $\leqslant 70 \%$ pred (mean FEV $1=45.8 \%$ pred) and 113 control subjects with an FEV1 of $>85 \%$. All subjects were followed from 1973-1974 to 1984. Lung cancer developed in nine subjects and two control subjects. Subjects with COPD had a four-times greater risk of developing lung cancer than those without airway obstruction when matched for age, sex, occupation, and smoking history. Similarly, Tockman et al. [7] demonstrated the risk of lung cancer in patients with 
Table 2. - Conditional logistic regression results

\begin{tabular}{|c|c|c|c|c|c|}
\hline & Controls & Cases & OR & $95 \% \mathrm{CI}$ & p-value \\
\hline Subjects n & 96 & 24 & & & \\
\hline \multicolumn{6}{|l|}{ Spirometry } \\
\hline FEV $1 \%$ pred & $75.6 \pm 17.6$ & $67.5 \pm 24.4$ & 1.2 & $1.0-1.5$ & 0.082 \\
\hline$\geqslant 81$ & $42(43.8)$ & $8(33.3)$ & 1.0 & & \\
\hline $61-80$ & $34(35.4)$ & $7(29.2)$ & 1.1 & $0.3-3.9$ & $>0.10$ \\
\hline $41-60$ & $17(17.7)$ & $4(16.7)$ & 1.2 & $0.3-4.7$ & $>0.10$ \\
\hline$\leqslant 40$ & $3(3.1)$ & $5(20.8)$ & 9.6 & $1.5-60.1$ & 0.016 \\
\hline $\mathrm{FEV} 1 / \mathrm{FVC} \times 100 \%$ & $64.4 \pm 10.2$ & $59.9 \pm 14.3$ & 1.4 & $1.0-2.2$ & 0.083 \\
\hline$\geqslant 71$ & $32(33.3)$ & $5(20.8)$ & 1.0 & & \\
\hline $61-70$ & $35(36.5)$ & $8(33.3)$ & 1.6 & $0.5-5.6$ & $>0.10$ \\
\hline $51-60$ & $17(17.7)$ & $4(16.7)$ & 1.7 & $0.4-7.4$ & $>0.10$ \\
\hline$\leqslant 50$ & $12(12.5)$ & $7(29.2)$ & 4.1 & $1.0-17.2$ & 0.056 \\
\hline \multicolumn{6}{|c|}{ Quantitative CT analysis } \\
\hline Emphysema \% & $9.9 \pm 8.6$ & $10.6 \pm 8.2$ & 1.1 & $0.6-1.9$ & $>0.10$ \\
\hline$<5$ & $36(37.5)$ & $9(37.5)$ & 1.0 & & \\
\hline $5-9$ & $18(18.8)$ & $1(4.2)$ & 0.2 & $0.0-2.0$ & $>0.10$ \\
\hline $10-14$ & $19(19.8)$ & $7(29.2)$ & 1.5 & $0.5-4.8$ & $>0.10$ \\
\hline$\geqslant 15$ & $23(24.0)$ & $7(29.2)$ & 1.1 & $0.4-3.7$ & $>0.10$ \\
\hline
\end{tabular}

Data are presented as $\mathrm{n}(\%)$ or mean \pm SD unless otherwise stated. OR: odds ratio; CI: confidence interval; FEV1: forced expiratory volume in one second; FVC: forced vital capacity; CT: computed tomography.

moderate to severe airway obstruction from the Intermittent Positive Pressure Breathing Trial $(n=676)$ and in participants with no obstruction to moderate obstruction in the Johns Hopkins Lung Project $(\mathrm{n}=$ 3,728 ). A total of 22 participants died of lung cancer in the Intermittent Positive Pressure Breathing Trial and 19 in the Johns Hopkins Lung Project. Patients with airway obstruction defined as an FEV1 of $<60 \%$ pred had a six-fold increased risk of lung cancer compared with those without airway obstruction. The risk of lung cancer increased with the severity of airway obstruction. Further evidence of the risk of lung cancer among those with airway obstruction was reported in the results of the Lung Health Study [23]. Among nearly 6,000 smokers with mild-to-moderate airway obstruction, lung cancer was the most common cause of death at the end of the $5 \mathrm{yrs}$ of follow-up.

The current study suggests that percentage of emphysema, as assessed by quantitative CT, is not associated with an increased risk of lung cancer after adjustment for sex, age and smoking history. The discrepancy in lung cancer risk between airway obstruction and percentage of emphysema in this study is consistent with the observation by GeLB et al. [24] that emphysema does not appear to be primarily responsible for airway obstruction in COPD. GELB et al. [24] found a fair to weak correlation between FEV1 \% pred and either CT or morphologically scored emphysema. Similar observations were noted by Gould et al. [25] and Kuwano et al. [26]. The discrepancy with previous reports $[27,28]$ which suggest that emphysema was primarily responsible for airway obstruction in COPD, may in part be related to limited availability of autopsy specimens of lobes or lungs [24].

Methodological limitations may provide another potential explanation for the results of this study. The percentage of emphysema was analysed by quantitative CT measurements of lung density using low-dose spiral CT. Quantitative CT with a standard-dose setting (140-200 mA) accurately reflects macroscopic emphysema [11]. Although reduction of the milliamperage increases the image noise, NAIDICH et al. [29] found that visualization of parenchymal structure was not affected by decreasing the milliamperage. ZWIREWICH et al. [30] showed that the low-dose and the standard-dose HRCT scans were equivalent in the evaluation of anatomical information as well as interstitial and air-space abnormalities. A recent study reported that low-dose spiral CT was equivalent to the standard-dose spiral CT at visualizing lung abnormalities [31]. Nevertheless, few studies have assessed emphysema by quantitating areas of low attenuation on low-dose spiral CT.

Another possible methodological limitation is the use of the lower attenuation threshold of $-900 \mathrm{HU}$ to identify emphysema in this study. The lower attenuation thresholds that have been used most widely are -900 or $-910 \mathrm{HU}$ on conventional 7 - to $10-\mathrm{mm}$ collimation CT [10-12, 15]. Using HRCT scans at 1-mm collimation, a lower attenuation threshold of $-950 \mathrm{HU}$ was found to correlate best with morphological emphysema [32]. However, the optimal threshold for the detection of emphysema on low-dose spiral CT has not been reported previously.

The authors acknowledge that the range of emphysema scores observed in the present study was smaller than would be expected. For the range of emphysema observed no compelling evidence was found to suggest that an increased percentage of emphysema was associated with an increased risk of lung cancer.

Although the results of this study suggest that percentage of emphysema based on quantitative CT measurements of lung density is not a significant risk factor for lung cancer after adjustment for sex, age and smoking history, several studies have suggested an increased risk of lung cancer associated with 
emphysema. Recently, a $2-5 \%$ rate of unsuspected lung cancer was found on CT scanning in patients with severe emphysema who were undergoing evaluation for possible lung-volume reduction surgery $[8,9]$. However, most of patients being evaluated for lung-volume reduction surgery have severe airway obstruction with an FEV1 of $\leqslant 40$ pred. On the basis of results from other studies [6,7], the higher risk of lung cancer in patients undergoing lung-volume reduction surgery is more likely to be attributable to the presence of severe airway obstruction rather than anatomical emphysema.

Epidemiological studies have also reported increased risks of lung cancer in relation to the previous diagnosis of lung diseases, such as emphysema, chronic bronchitis and bronchial asthma $[33,34]$. MAYNE et al. [33] conducted a population-based case-controlled study of lung cancer in male and female nonsmokers in New York. Statistically significant associations were found for previous history of emphysema $(\mathrm{OR}=1.94$, 95\% CI 1.10-3.43), chronic bronchitis (OR $=1.73,95 \%$ CI 1.10-2.72) and bronchial asthma (OR=1.82, 95\% CI 1.26-2.63). BROWNSON et al. [34] also found a history of emphysema to be associated with an increased risk of lung cancer among females in Missouri $(\mathrm{OR}=2.7$, 95\% CI 1.8-4.2). However, the history of physiciandiagnosed previous lung disease in these studies was based on self-report and therefore recall bias is a major concern [33, 34]. Another concern is that there may be misclassification among reported emphysema, chronic bronchitis and bronchial asthma.

Several mechanisms have been suggested to explain the predisposition of patients with COPD to developing lung cancer. Mucociliary clearance is impaired with COPD [35, 36]. During the clearing process, particles tend to pool in areas with impaired mucociliary clearance. This pooling may allow carcinogens from the smoke in the mucous blanket to have longer exposure time at these sites, leading to development of lung cancer [6]. CoHEN et al. [37] have described familial clustering of pulmonary dysfunction in relatives of patients with lung cancer as well as in patients with COPD, suggesting that lung cancer and COPD share a common familial predisposition. There is increasing interest in whether carriers of an $\alpha_{1}$ antitrypsin deficiency are at increased risk of lung cancer [34]. $\alpha_{1}$-Antitrypsin deficiency accounts for $<1 \%$ of COPD in the USA [3]. Although there are no reports of an increased risk of lung cancer in patients with homozygous $\alpha_{1}$-antitrypsin deficiency, YANG et al. [38] recently demonstrated that lung cancer patients are significantly more likely to carry the mutated $\alpha_{1}$-antitrypsin allele than the general population.

To conclude, the results of this study support the previous observation of an increased risk of lung cancer associated with airway obstruction, particularly severe obstruction. In addition, no compelling evidence was found to suggest that an increased percentage of emphysema, as assessed by quantitative computed tomography analysis using low-dose spiral computed tomography, is associated with lung cancer risk.

\section{References}

1. Greenlee RT, Murray T, Bolden S, Wingo PA. Cancer statistics 2000. CA Cancer J Clin 2000; 50: 7-33.

2. American Thoracic Society Statement. Cigarette smoking and health. Am J Respir Crit Care Med 1996; 153: 861-865.

3. American Thoracic Society. Standards for diagnosis and care of patients with chronic obstructive pulmonary diseases. Am J Respir Crit Care Med 1995; 152: S77-S121.

4. US Surgeon General. The health consequence of smoking: chronic obstructive lung disease. DLIHS Publication No.84-50205. Washington, DC. US Department of Health and Human Services. 1984.

5. Davis AL. Bronchogenic carcinoma in chronic obstructive pulmonary disease. JAMA 1976; 235: 621-622.

6. Skillrud DM, Offord KP, Miller RD. Higher risk of lung cancer in chronic obstructive pulmonary disease. A prospective, matched, controlled study. Ann Int Med 1986; 106: 503-507.

7. Tockman MS, Anthonisen NR, Wright EC, Donithan MG. The intermittent positive pressure breathing trial group, The Johns Hopkins lung project for the early detection of lung cancer. Airway obstruction and the risk for lung cancer. Ann Int Med 1987; 106: 512-518.

8. McKenna RJ, Fischel RJ, Brenner M, Gelb AF. Combined operation for lung volume reduction surgery and lung cancer. Chest 1996; 110: 885-888.

9. Rozenshtein A, White CS, Austin JHM, Roinney BM, Protopapas Z, Krasna MJ. Incidental lung carcinoma detected at $\mathrm{CT}$ in patients selected for lung volume reduction surgery to treat severe pulmonary emphysema. Radiology 1998; 207: 487-490.

10. Stern EJ, Frank MS. CT of the lung in patients with pulmonary emphysema: Diagnosis, quantification, and correlation with pathologic and physiologic findings. AJR 1994; 162: 791-798.

11. Muller NL, Staples CA, Miller RR, Abboud RT. "Density Mask" An objective method to quantitate emphysema using, computed tomography. Chest 1988; 94: 782-787.

12. Gurney JW, Jones KK, Robbins RA, et al. Regional distribution of emphysema: correlation of highresolution CT with pulmonary function tests in unselected smokers. Radiology 1992; 183: 457-463.

13. Adams H, Bernard MS, McConnochic K. An appraisal of CT pulmonary density mapping in normal subjects. Clin Radiol 1991; 43: 238-242.

14. Hartman TE, Tazelaar HD, Swensen SJ, Muller NL. Cigarette smoking: CT and pathologic findings of associated pulmonary diseases. Radiographics 1997; 17: 377-390.

15. Kauczor HU, Heussel CP, Fischer B, Klamm R, Mildenberger $\mathrm{P}$, Thelen $\mathrm{M}$. Assessment of lung volume using helical $\mathrm{CT}$ at inspiration and expiration: Comparison with pulmonary function tests. $A J R$ 1998; 171: 1091-1095.

16. Jett JR. Screening for lung cancer in high-risk groups: current status of low-dose spiral CT scanning and sputum markers. Sem Respir Crit Care Med 2000; 21: 385-392.

17. Rosenbaum PR. Optimal matching for observational studies. JAMA 1989; 84: 1024-1032.

18. Bergstralh EJ, Kosanke JL. Computerized matching of controls. Section of Biostatics, Technical Report 56. Rochester, MN, USA. Mayo Foundation, 1995. 
19. Standardization of spirometry: 1987 update-statement of the American Thoracic Society. Am Rev Respir Dis 1987; 136: 1285-1298.

20. Crapo RO, Morris AH, Gardner RM. Reference spirometric values using techniques, and equipment that meet ATS recommendations. Am Rev Respir Dis 1981; 123: 659-664.

21. Breslow NE, Day NE. Statistical methods in cancer research, volume 1-the analysis of case-control studies. Lyon. IARC Scientific Publication 32, 1980.

22. American Thoracic Society. Medical section of the American Lung Association. Evaluation of impairment/disability secondary to respiratory disorders. Am Rev Respir Dis 1986; 133: 1205-1209.

23. Anthonisen NR, Connett JE, Killey JP, et al. Effects of smoking intervention and the use of inhaled anticholinergic bronchodilator on the rate of decline in FEV1: Lung health study. JAMA 1994; 272: $1497-$ 1505.

24. Gelb AF, Houg JC, Maller NL, et al. Contribution of emphysema and small airways in COPD. Chest 1996; 109: 353-359.

25. Gould GA, Macnee W, Mclean A, et al. CT measurement of lung density in life can quantitate distal airspace enlargement: An essential defining feature of human emphysema. Am Rev Respir Dis 1988; 137: 380-392.

26. Kuwano K, Matsuba K, lkeda T, et al. The diagnosis of mild emphysema: correlation of computed tomography and pathology scores. Am Rev Respir Dis 1990; 141: 169-178.

27. Hale KA, Ewing SL, Gosnell BA, Niewoehner DE. Lung disease in long term cigarette smokers with and without chronic airflow obstruction. Am Rev Respir Dis 1984; 130: 716-721.

28. Nagai A, West WW, Thurbeck WM. The national institute of health intermittent positive pressure breathing trial: pathology studies: II. Correlation between morphologic findings, clinical findings and evidence of expiratory airflow obstruction. Am Rev Respir Dis 1985; 132: 946-953.

29. Naidich DP, Marshall CH, Gribbin G, Arams RS, McCauley DI. Low-dose CT of the lungs: preliminary observations. Radiology 1990; 175: 729-731.

30. Zwirewich CV, Mayo JR, Muller NL. Low-dose highresolution CT of lung parenchyma. Radiology 1991; 180: 413-417.

31. Takahashi M, Maguire WM, Ashtari M, et al. Lowdose spiral computed tomography of the thorax. Comparison with the standard-dose technique. Invest Radiol 1998; 33: 68-73.

32. Gevenois PA, de Maertelaer V, Du Vuyst P, Zanen J, Yernault X. Comparison of computed density and macroscopic morphometry in pulmonary emphysema. Am J Respir Care Med 1995; 152: 653-657.

33. Mayne ST, Buenconsejo J, Janerich DT. Previous lung disease and risk of lung cancer among men and women nonsmokers. Am J Epidemiol 1999; 149: 13-20.

34. Brownson RC, Alavanja MCR. Previous lung disease and lung cancer risk among women (United States). Cancer Causes Control 2000; 11: 853-858.

35. Lourenço RV, Loddenkemper R, Carton RW. Pattern of distribution and clearance of aerosol in patients with bronchiectasis. Am Rev Respir Dis 1972; 106: 857-866.

36. Lourenço RV, Klimek MF, Borowski CI. Deposition and clearance of 2-E particles in the trachobronchial tree of normal subjects-smokers and nonsmokers. $J$ Clin Invest 1971; 50: 1411-1420.

37. Cohen BH, Graves CG, Uvy DA, et al. A common familial component in lung cancer and chronic obstructive pulmonary disease. Lancet 1977; 2: $523-$ 536.

38. Yang P, Wentzlaff KA, Katzmann JA, et al. Alpha 1-antitrypsin deficiency allele carriers among lung cancer patients. Cancer Epidemiol Biomakers Prev 1999; 8: 461-465. 